\title{
LETTERS
}

\section{To what end, evolutionary medicine?}

I read with great interest the article by Dr. Brunham that recommends a conceptual change in how we see medicine, from a mechanistic view to an evolutionary view. ${ }^{1}$ Although mostly descriptive, the final section suggests major changes to medical education and research funding, and summarizes by stating: "Evolution focuses on genes and clinicians focus on patients. Health is not directly selected by natural selection; rather, it is an indirect effect of organisms selected for reproductive success. Health is the central goal of medicine."

Two comments come to mind. One relates to the way in which natural selection is typically understood - that certain traits (or genes, simply put) provide a selective "advantage" in the context of survival of the fittest. I believe it is more accurate to say that certain traits (or genes) exist because they ended up being passed on by previous generations. The difference is important, particularly for a proposal to view medicine through an evolutionary lens. If evolution is forward- looking, then all kinds of interventions to change its course might be reasonable. If evolution, instead, truly exists only in the past tense, insofar as selective advantages are only real in retrospect, then evolutionary medicine is open to the same critique of being somewhat nihilistic as is the evolutionary perspective in general.

The second comment is related. If evolutionary natural selection inexorably works on patients and their children for all future generations, in all the complex interactions between each of our individual environments/genomes and epigenomes/ microbiomes and so on, with the end result that future patients have certain genes because of that process of natural selection - on what basis do we practise medicine that cares for the weak and wounded, or those past their reproductive stage? Although evolutionary medicine may offer a uniquely powerful paradigm for why current diseases exist, its logical application (if any) to the practice of medicine would seem to be to focus resources on our healthiest patients in their pre- and reproductive years, and withdraw resources from those who are old or infirm, or with mental health problems or genetic abnormalities, for example. This view of medicine is at distinct odds (apart from notable exceptions) with that promoted throughout human history, in which the old, infirm and unwell were cared for simply because they were old, infirm or unwell.

As Dr. Brunham notes, with superb understatement, "Applying evolutionary medicine to clinical practice will not be straightforward." ${ }^{1}$ The claim that an evolutionary medicine viewpoint "does provide a secure foundation for future developments in medicine"1 may depend more on your interpretation of what medicine ought to be than the merits of the viewpoint itself.

\section{Noel Corser MD}

Family physician, Hinton, Alta.

Cite as: CMAJ 2018 October 1;190:

E1173. doi: $10.1503 / \mathrm{cmaj} .70086$

\section{Reference}

1. Brunham RC. The genome, microbiome and evolutionary medicine. CMAJ 2018;190:E162-6.

Competing interests: None declared. 